\title{
Alkenenitriles: Zn-Cu Promoted Conjugate Additions of Alkyl Iodides in Water
}

Fraser F. Fleming* and Subrahmanyam Gudipati

Department of Chemistry and Biochemistry, Duquesne University, Pittsburgh PA 15282-1530

Supporting Information Table of Contents

\begin{tabular}{lcc}
\hline Compound & ${ }^{\mathbf{1}} \mathbf{H}$ NMR & ${ }^{\mathbf{1 3}} \mathbf{C}$ NMR \\
\hline $\mathbf{7 a}$ & S2 & S3 \\
$\mathbf{7 b}$ & $\mathrm{S} 4$ & S5 \\
$\mathbf{7 c}$ & $\mathrm{S} 6$ & $\mathrm{~S} 7$ \\
$\mathbf{7 d}$ & $\mathrm{S} 8$ & $\mathrm{~S} 9$ \\
$\mathbf{7 e}$ & $\mathrm{S} 10$ & $\mathrm{~S} 11$ \\
$\mathbf{7 f}$ & $\mathrm{S} 12$ & $\mathrm{~S} 13$ \\
$\mathbf{7 g}$ & $\mathrm{S} 14$ & $\mathrm{~S} 15$ \\
$\mathbf{7 h}$ & $\mathrm{S} 16$ & $\mathrm{~S} 17$ \\
$\mathbf{9 b}$ & $\mathrm{S} 18$ & $\mathrm{~S} 19$ \\
$\mathbf{9 d}$ & $\mathrm{S} 20$ & $\mathrm{~S} 21$ \\
\hline
\end{tabular}

General Procedure: Water (192 equiv) was added to a stirred mixture of Zn dust (3 equiv, 100 mesh) and powdered CuI (0.5 equiv). After 10 min silica gel (27 equiv, 230-400 mesh) was added, followed by additional water (192 equiv) and the alkenenitrile (1 equiv). After 15 min. the alkyliodide (1 equiv) was added, followed by 8 sequential additions of the iodide (1 equiv) at $1 \mathrm{~h}$ intervals. Two additional infusions of Zn (3 equiv) and CuI (0.5 equiv) were added after $3 \mathrm{~h}$ and 6 h with additional water (192 equiv) added during the last addition. The resulting mixture was stirred overnight, filtered through a glass fritted funnel, and then the crude product was extracted with EtOAc, dried $\left(\mathrm{Na}_{2} \mathrm{SO}_{4}\right)$, concentrated, and purified by radial chromatography (1:20 EtOAc/hexanes) to afford analytically pure material. 


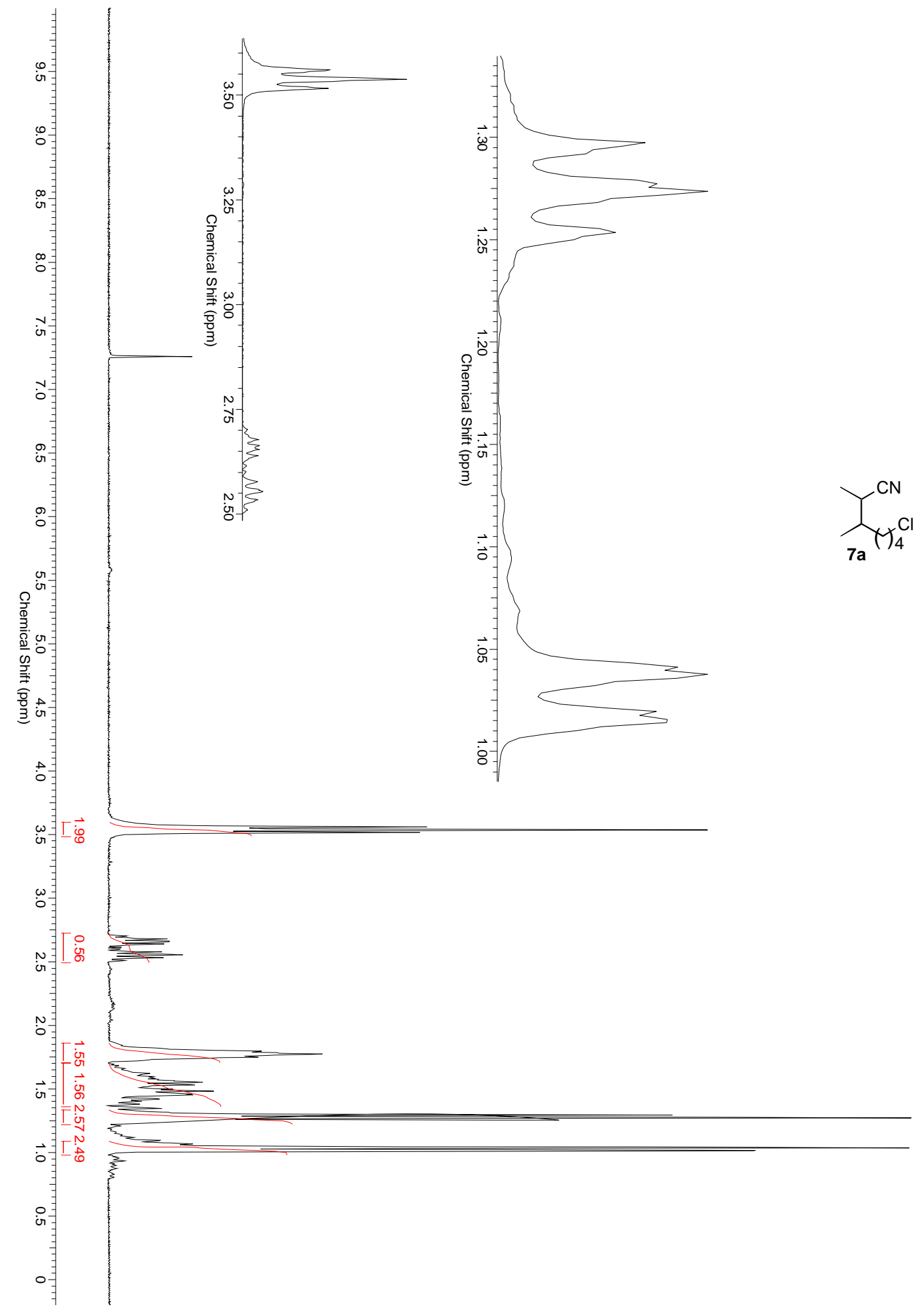




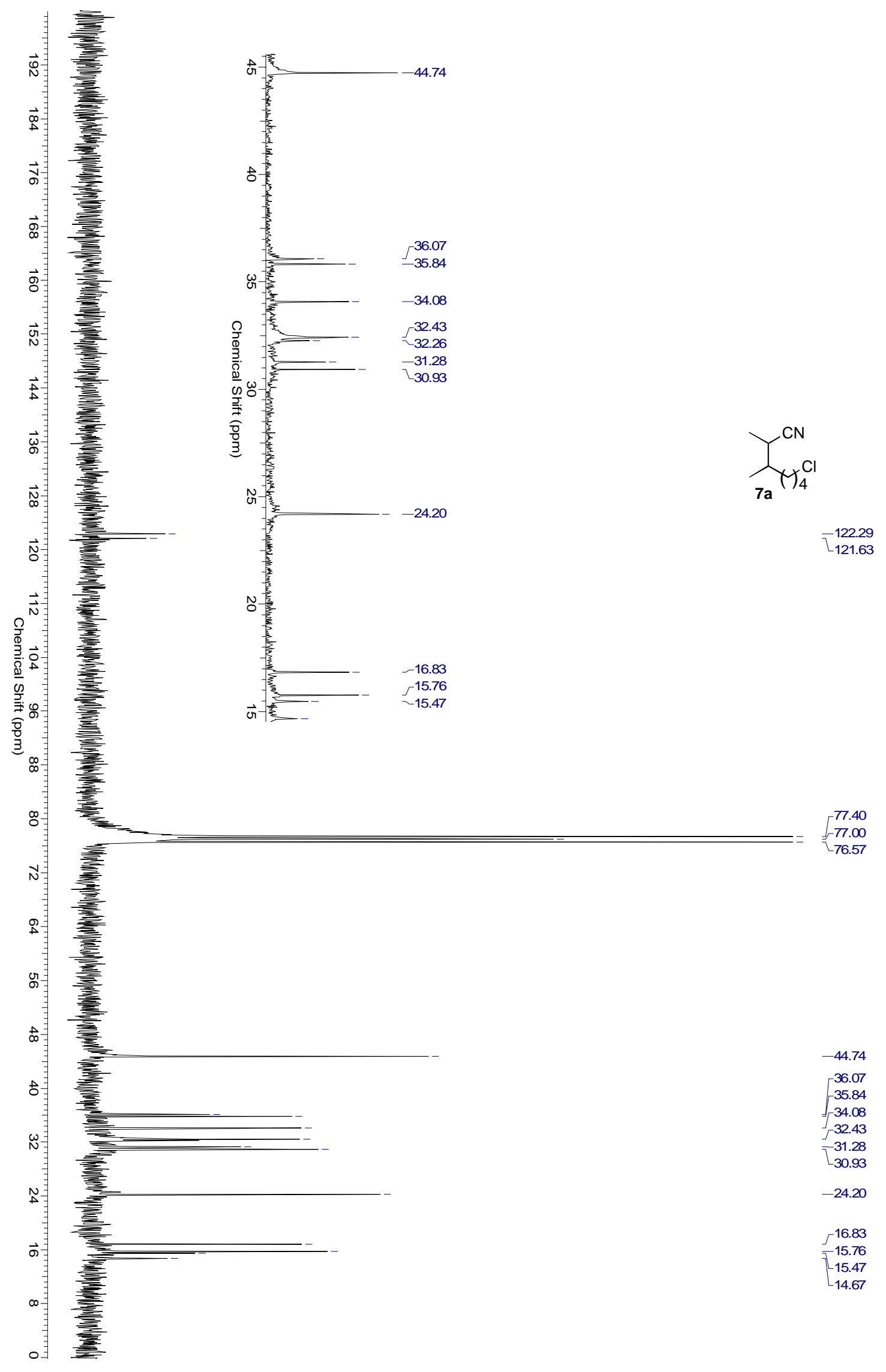




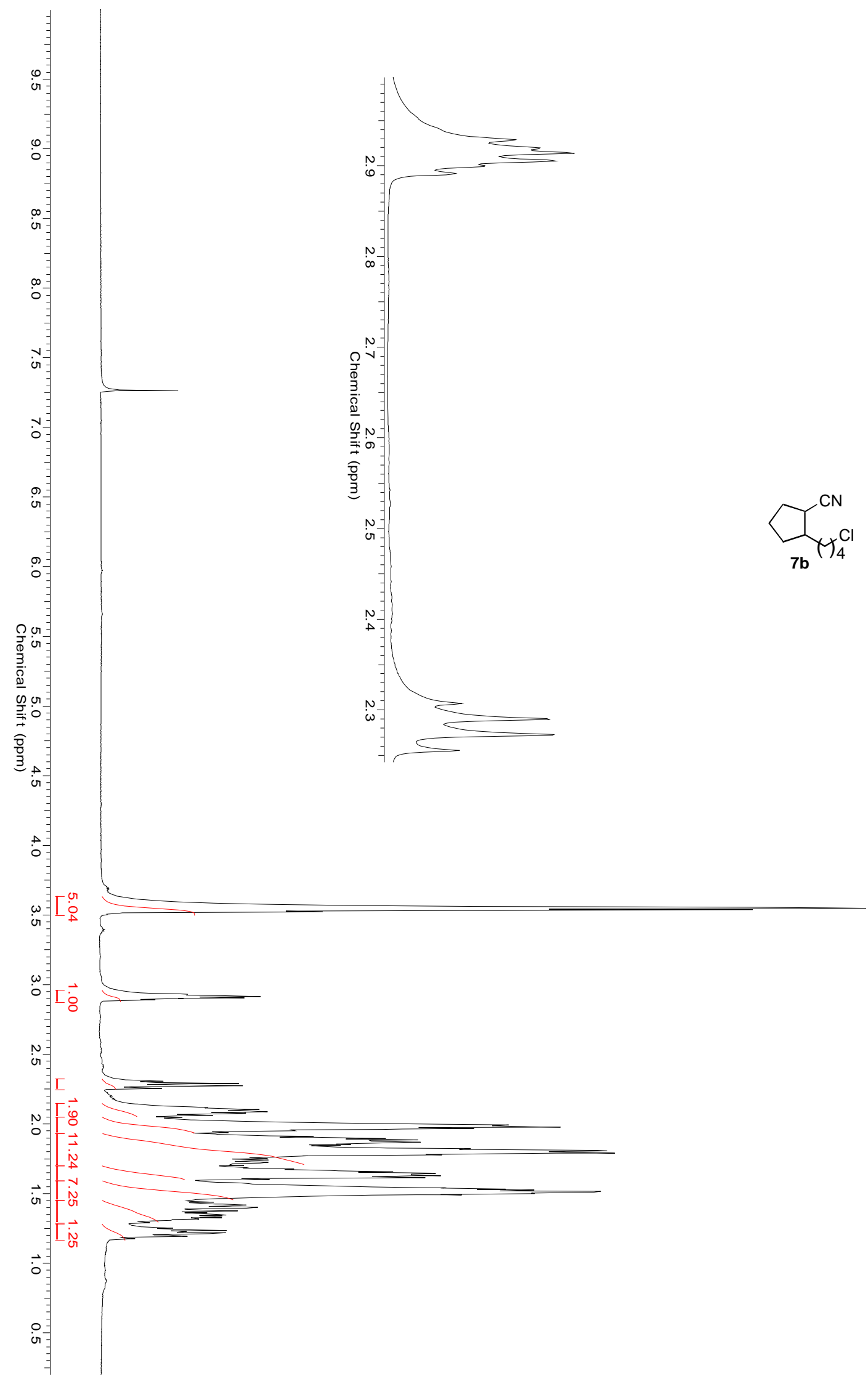




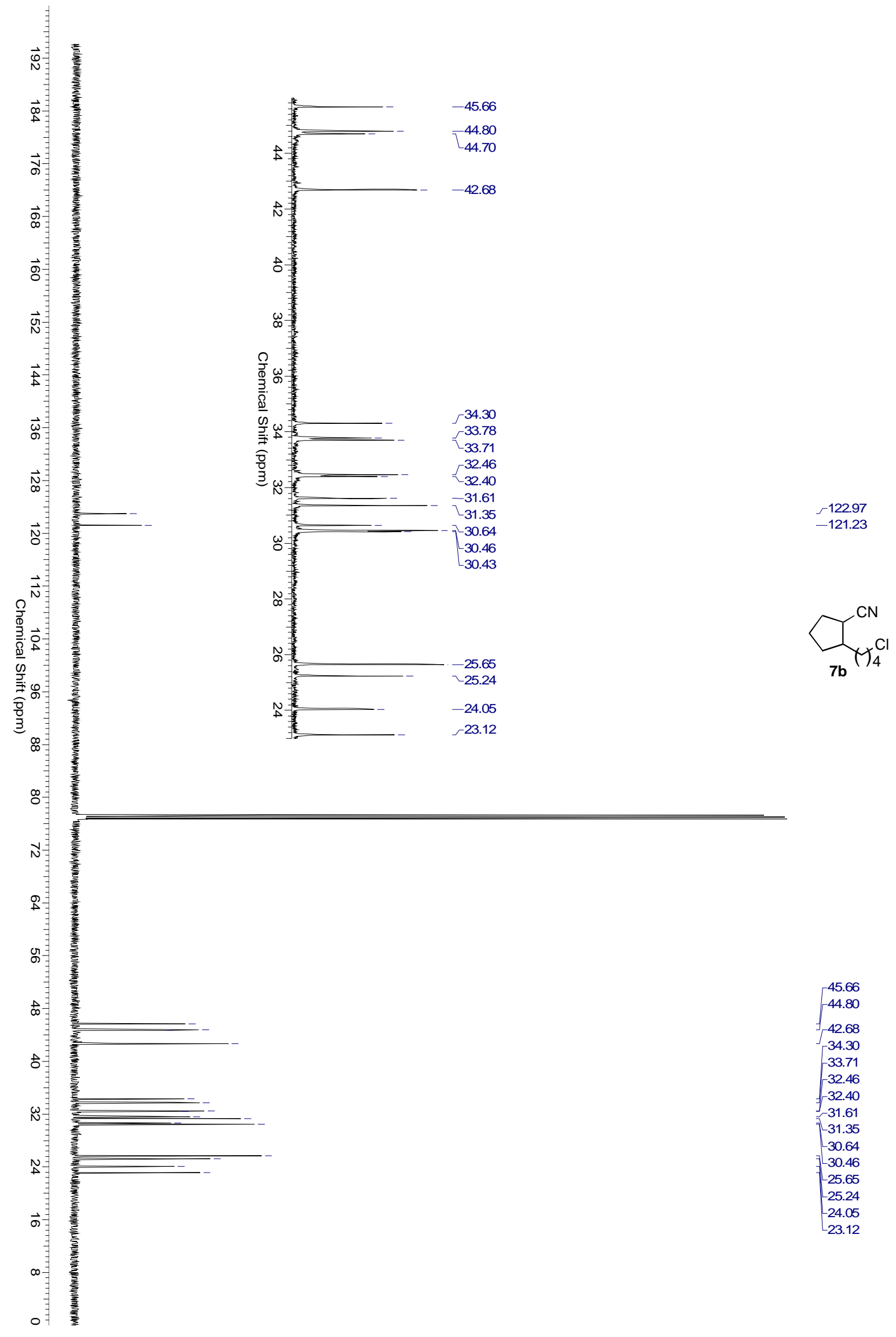




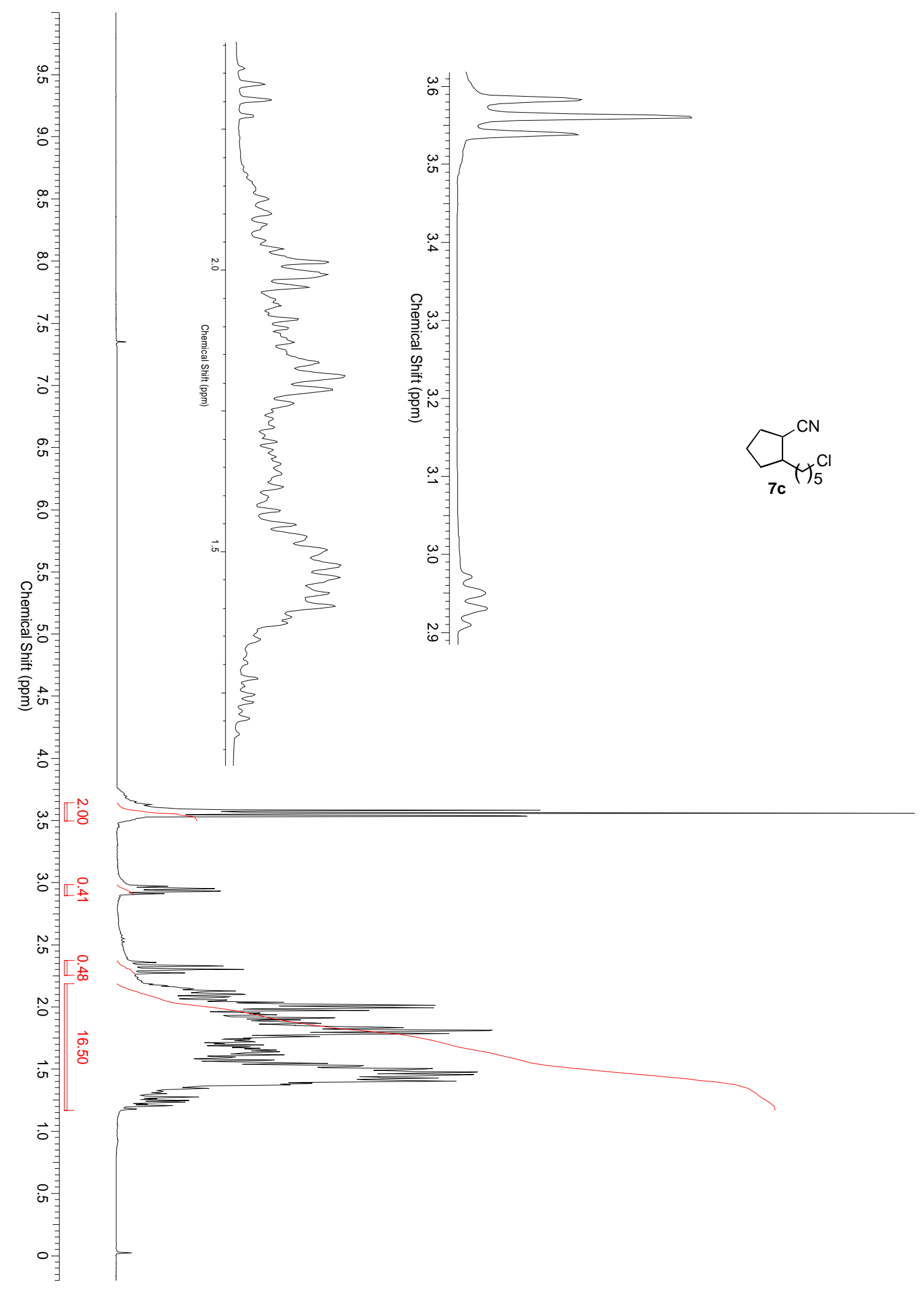




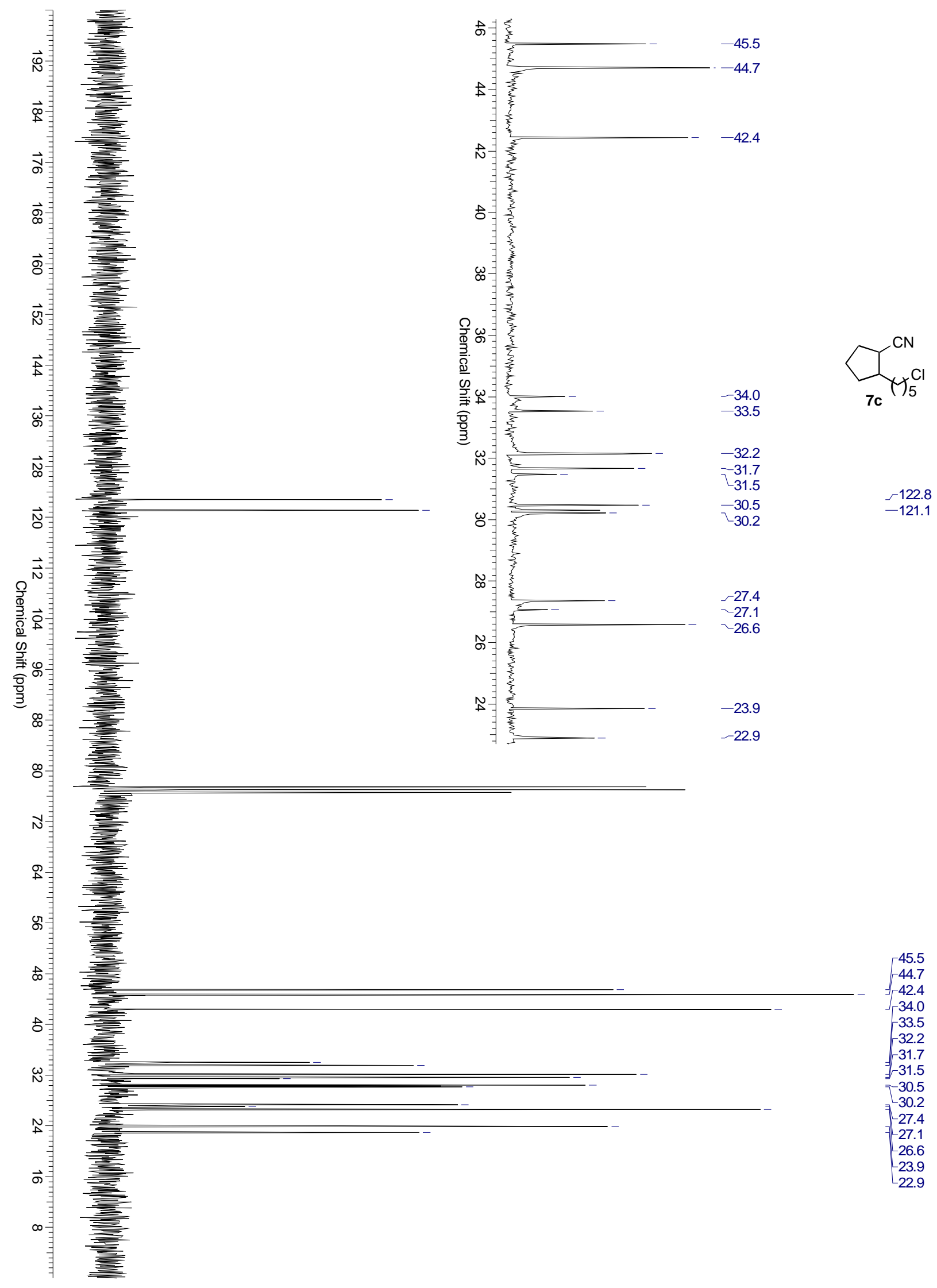




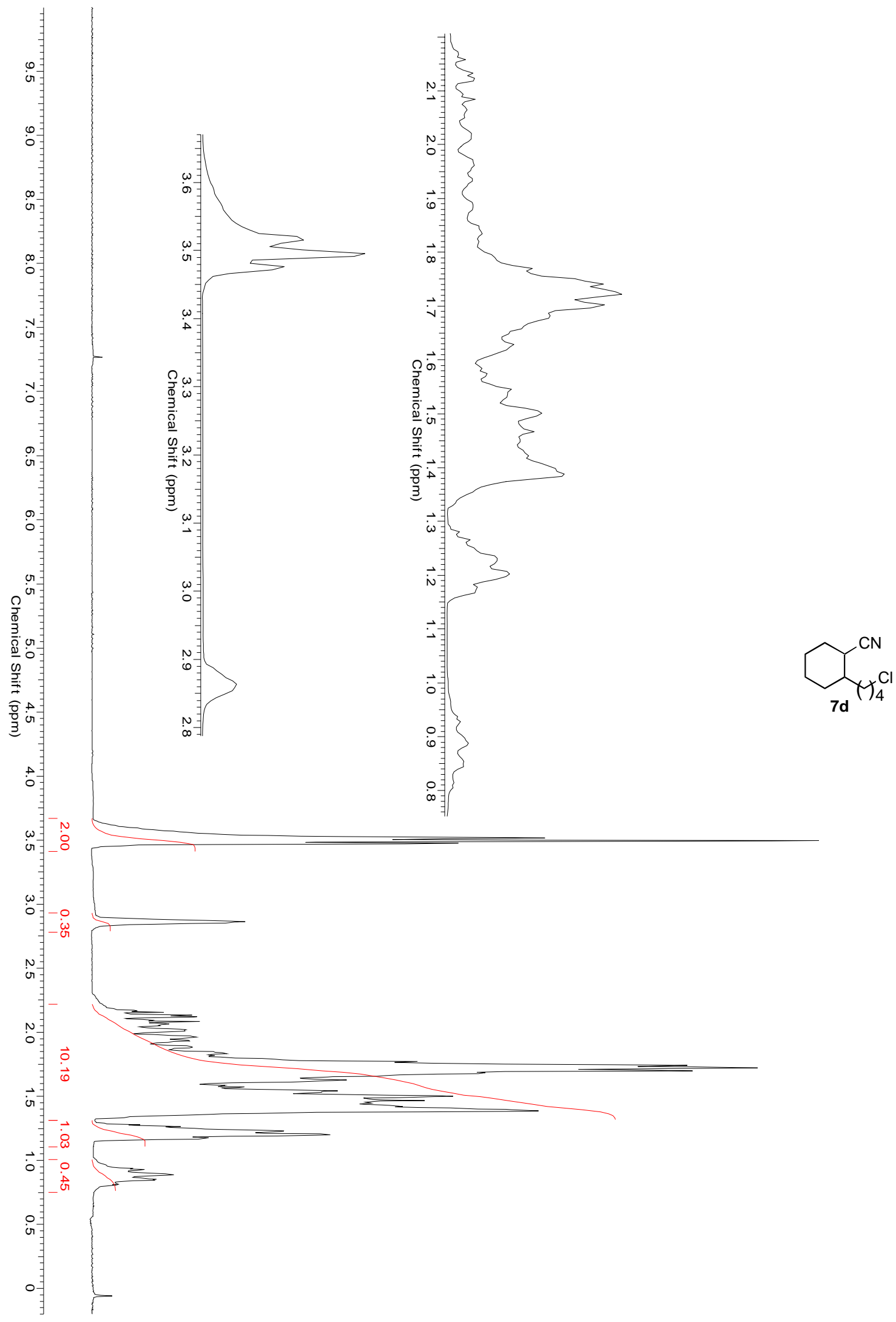




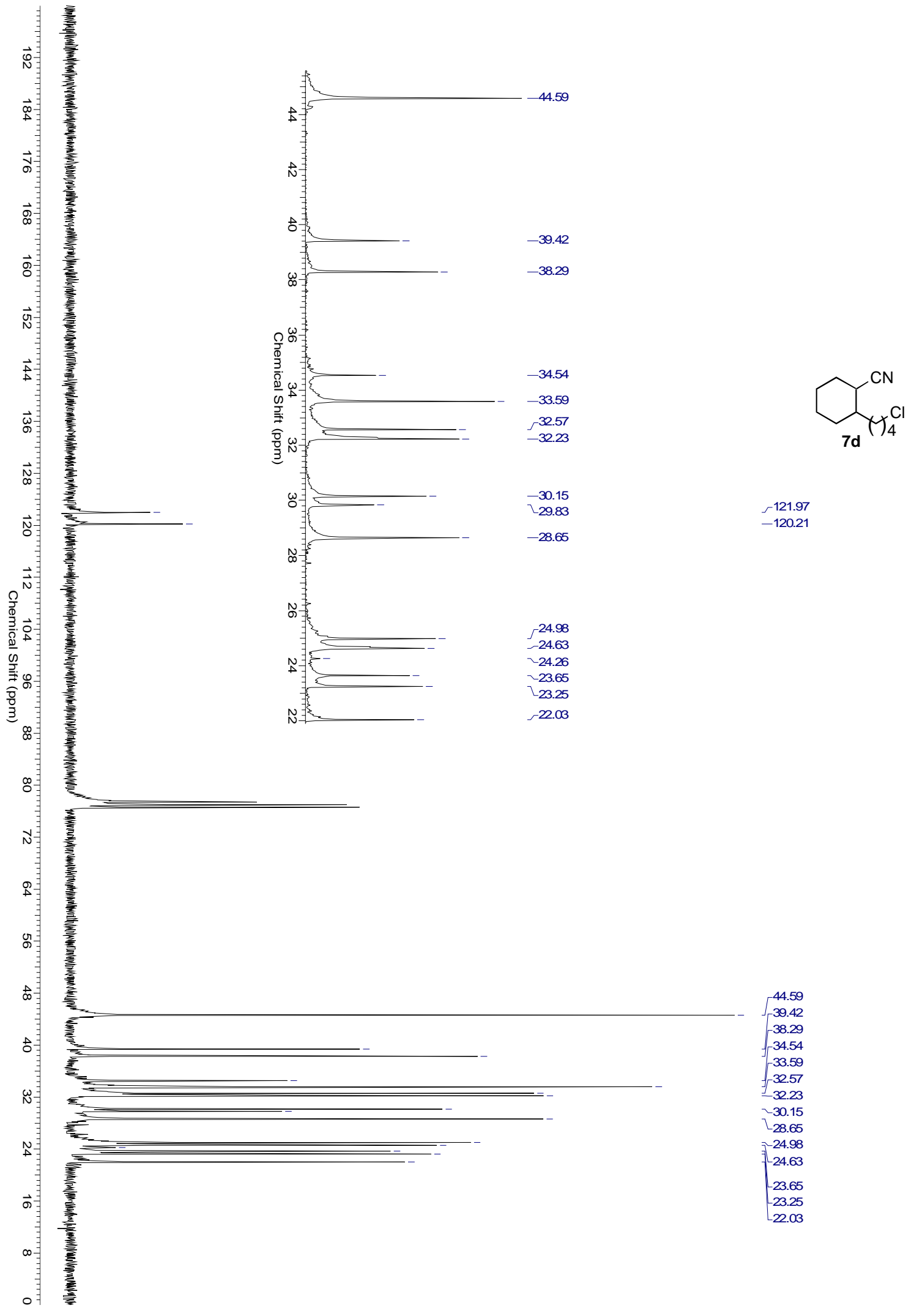




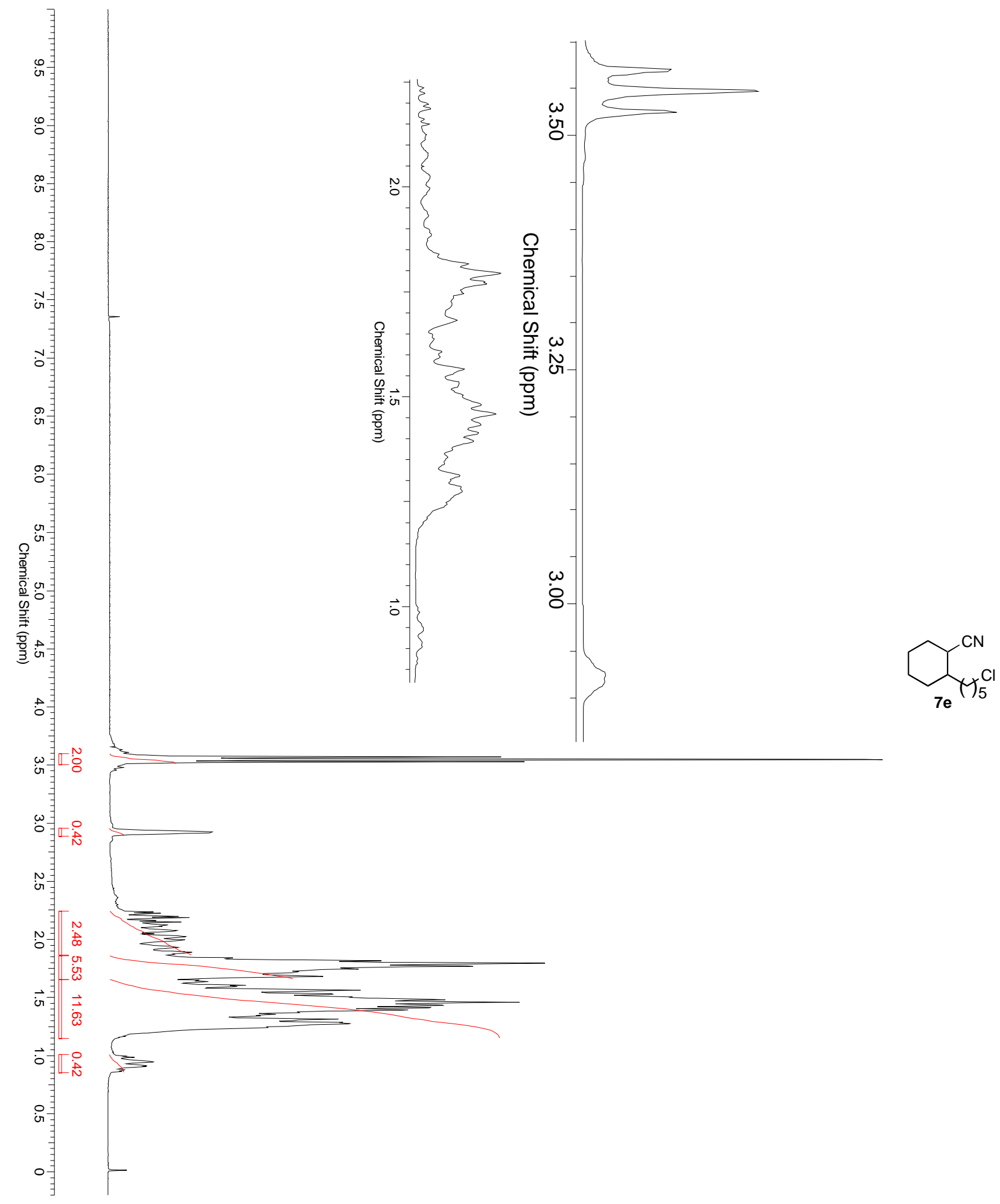




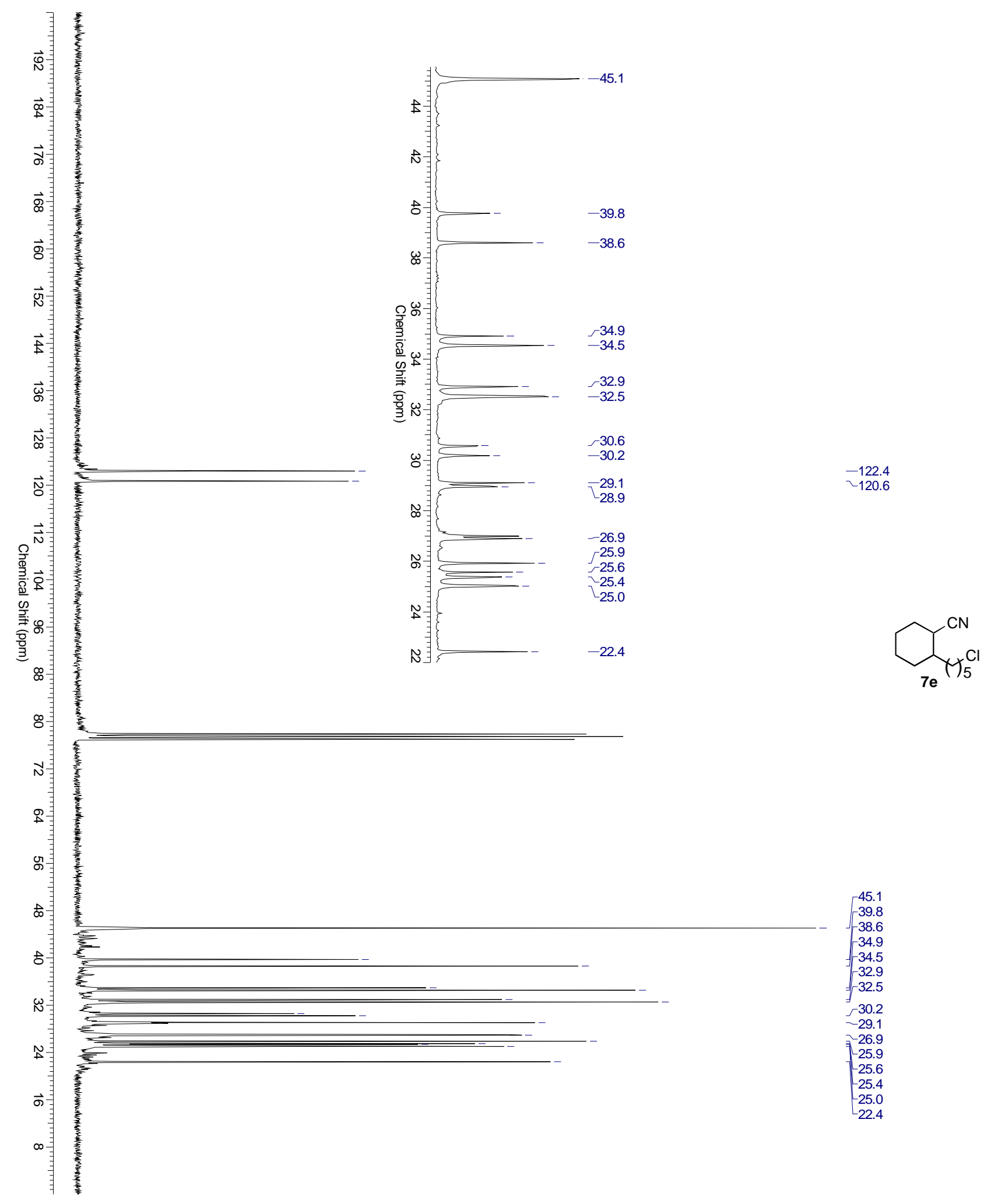




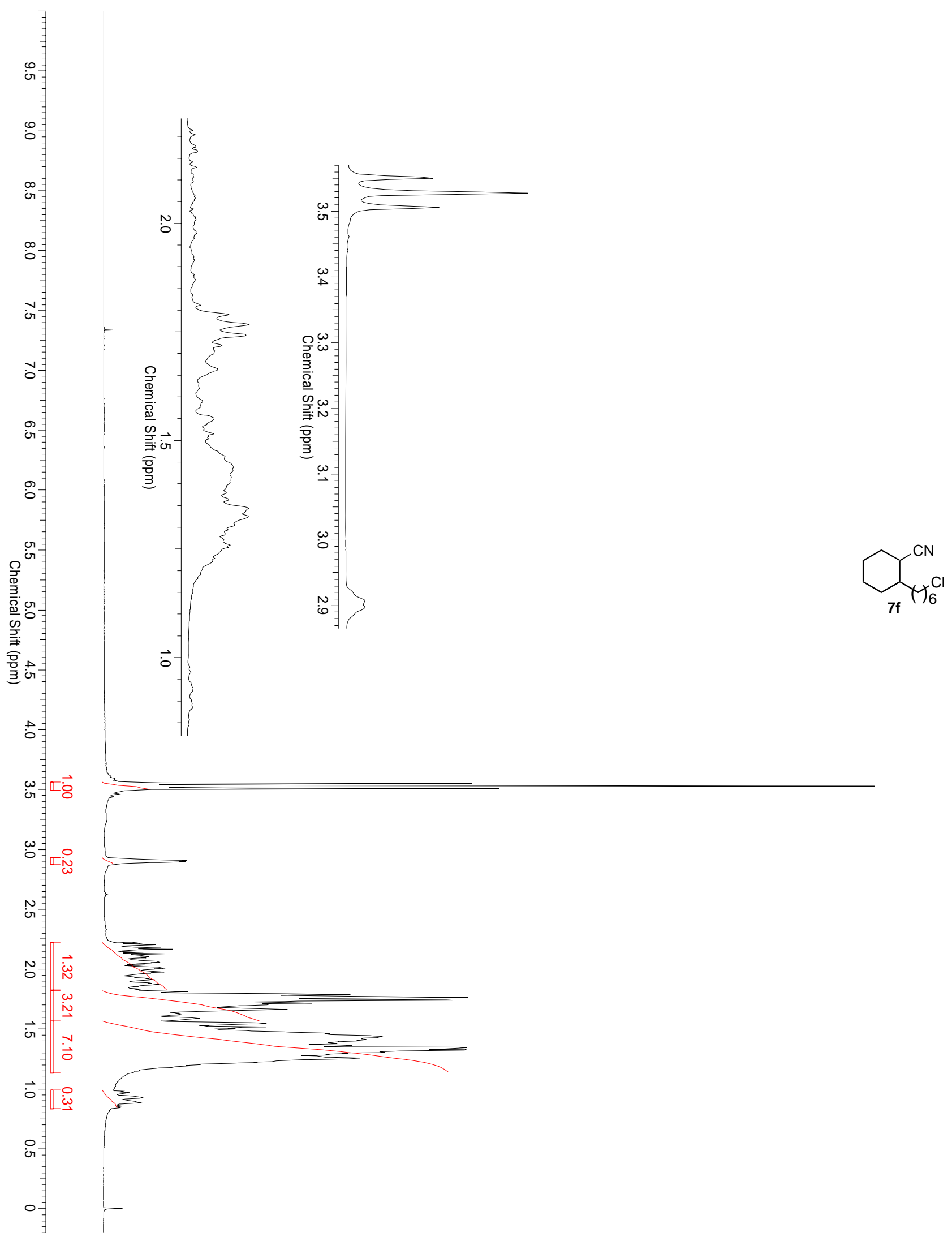




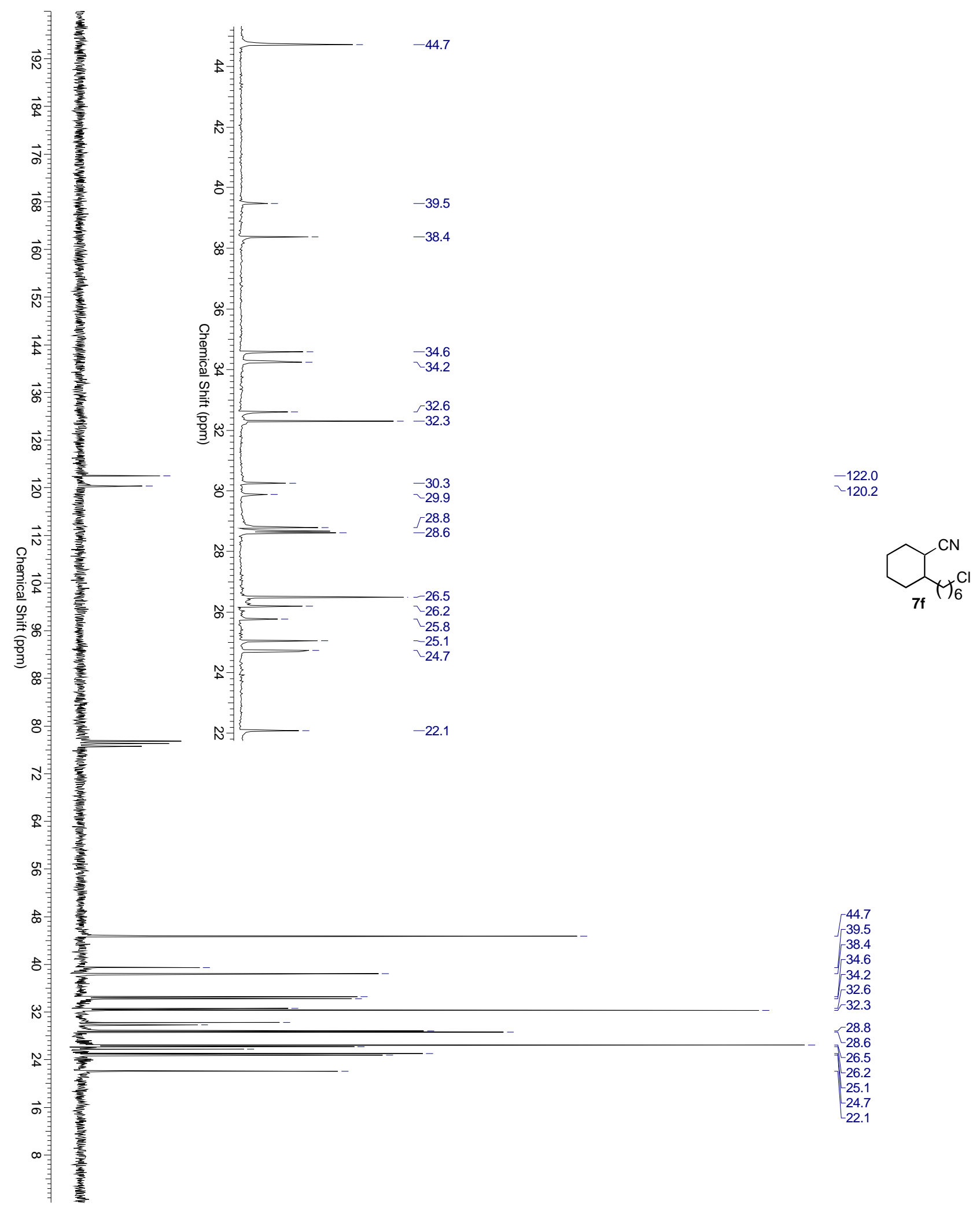




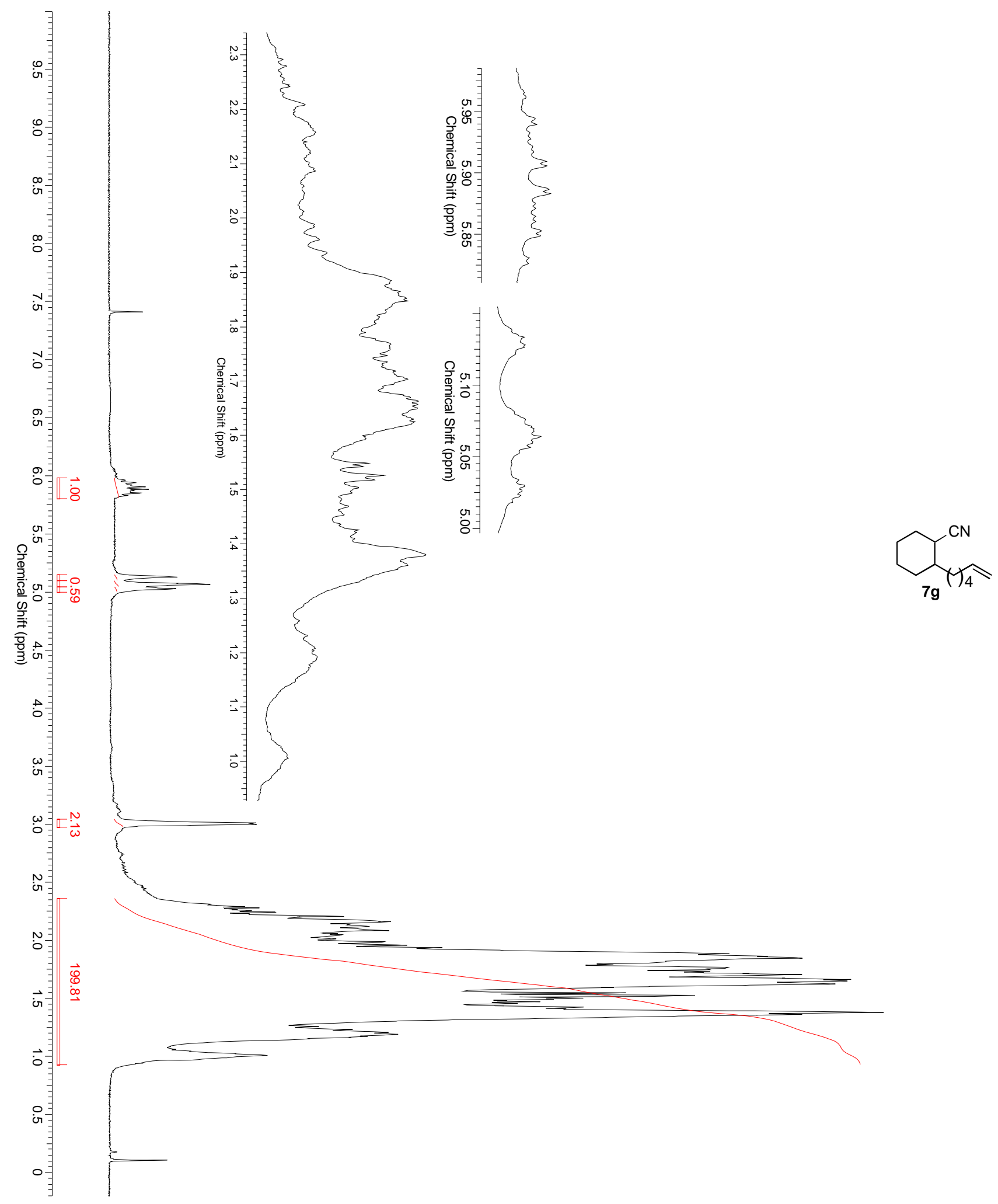




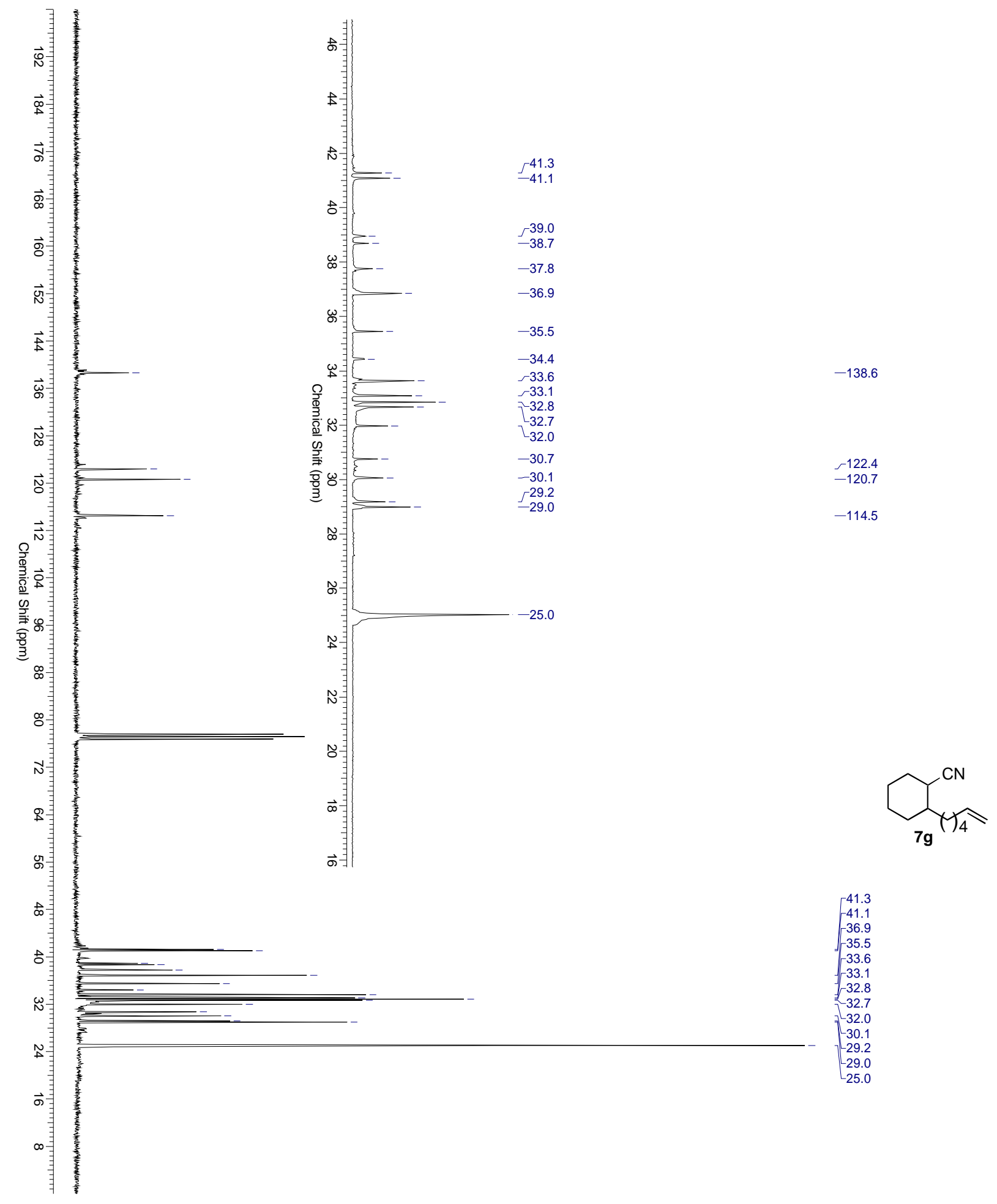




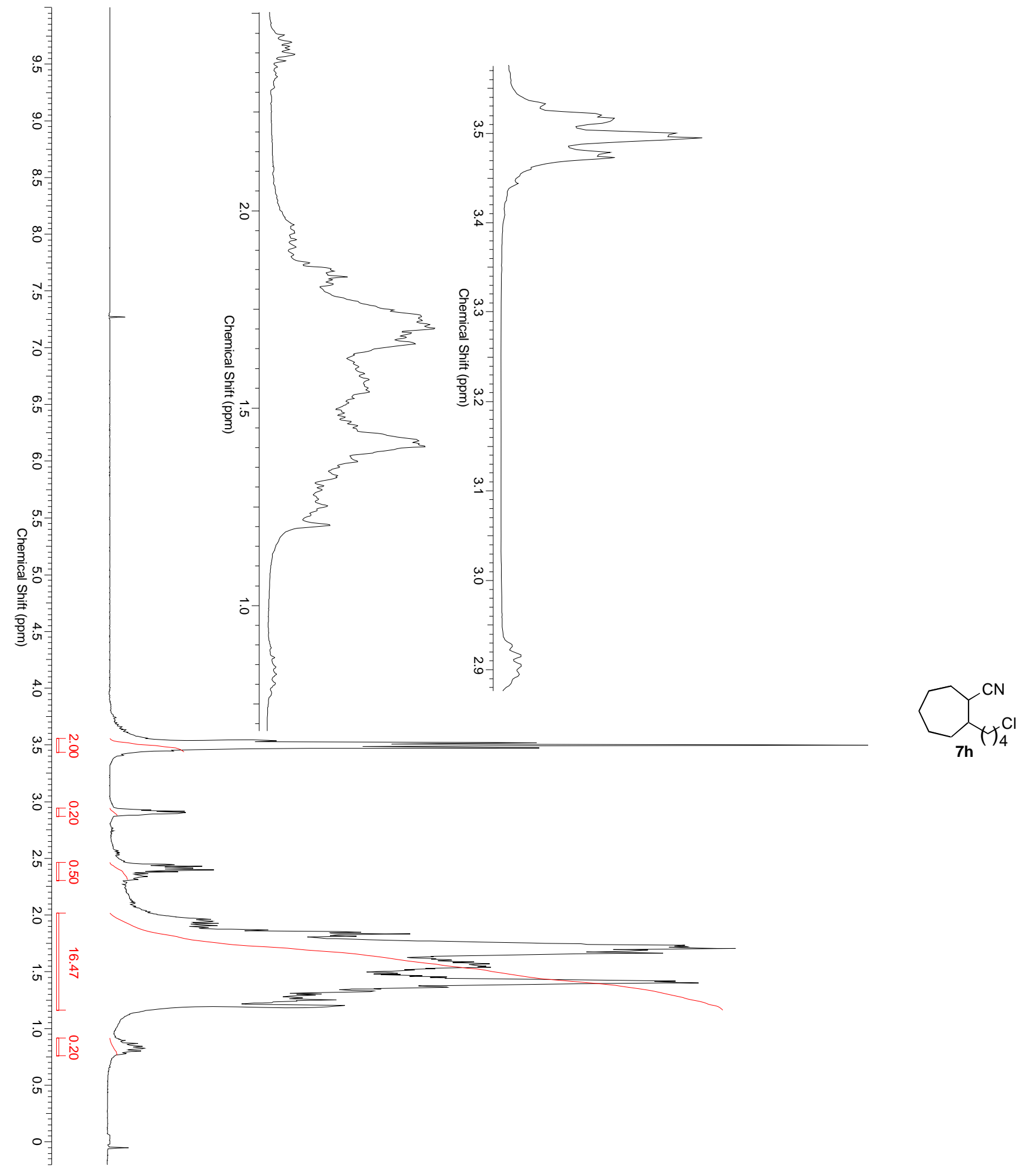




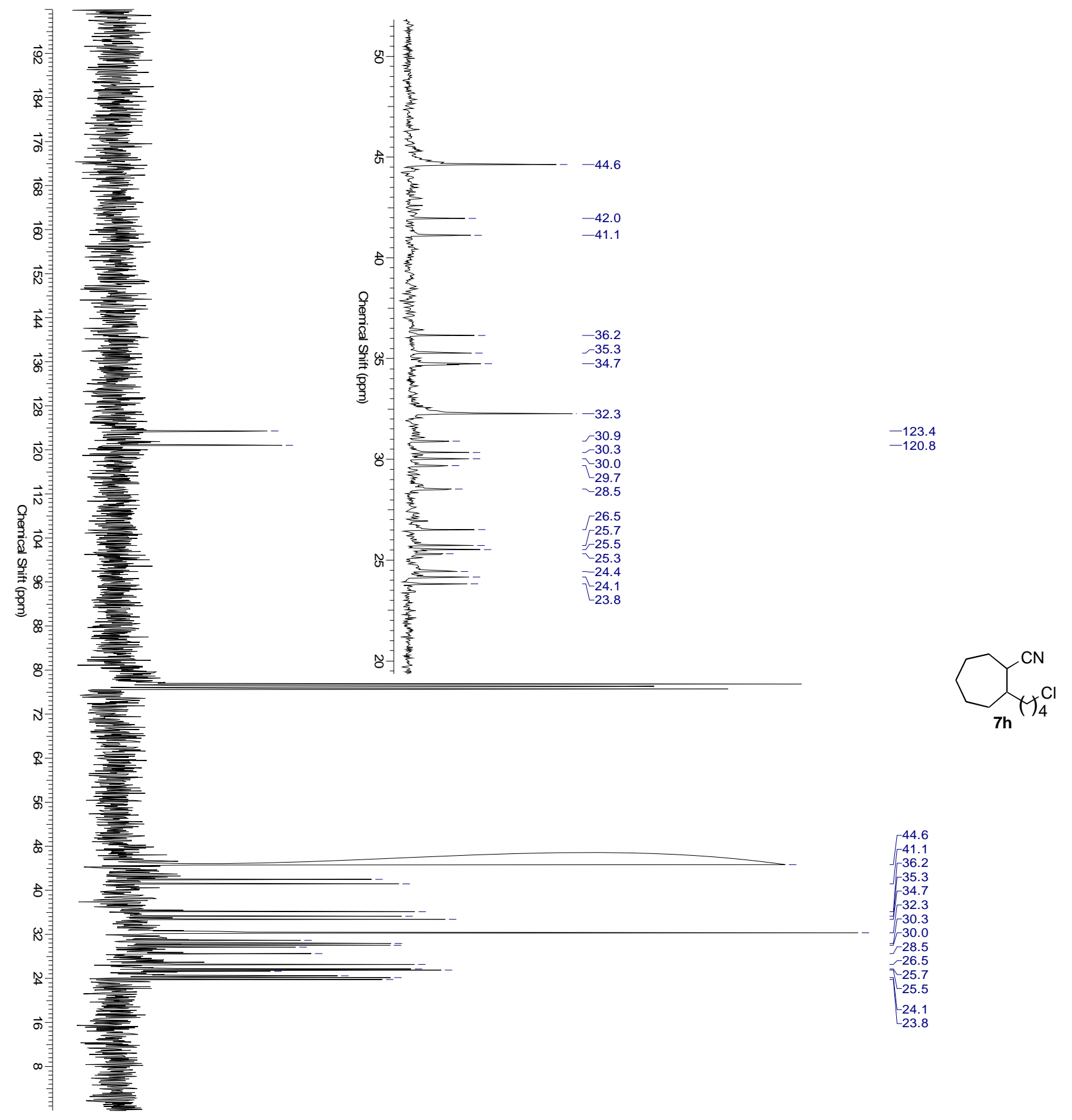




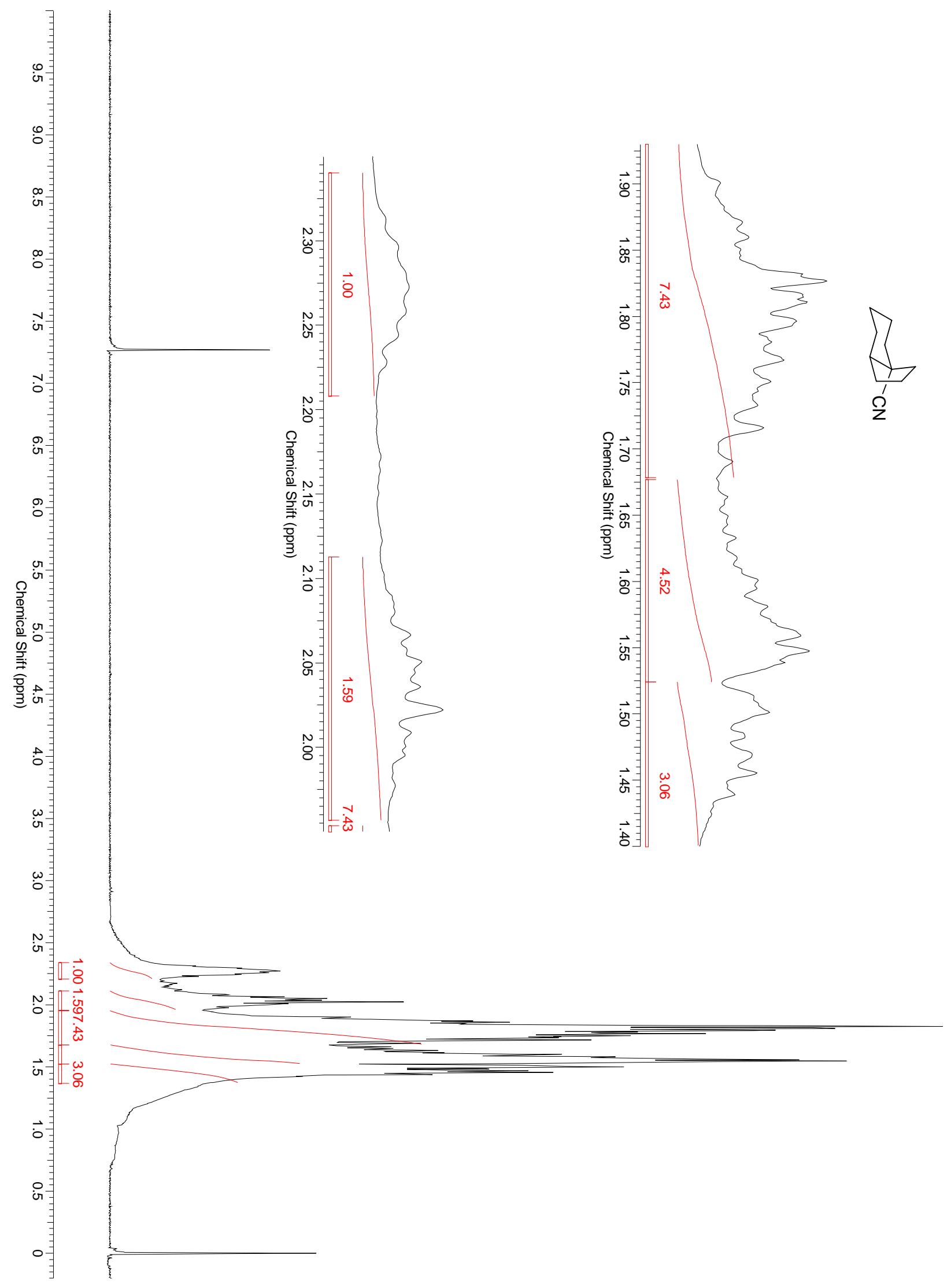




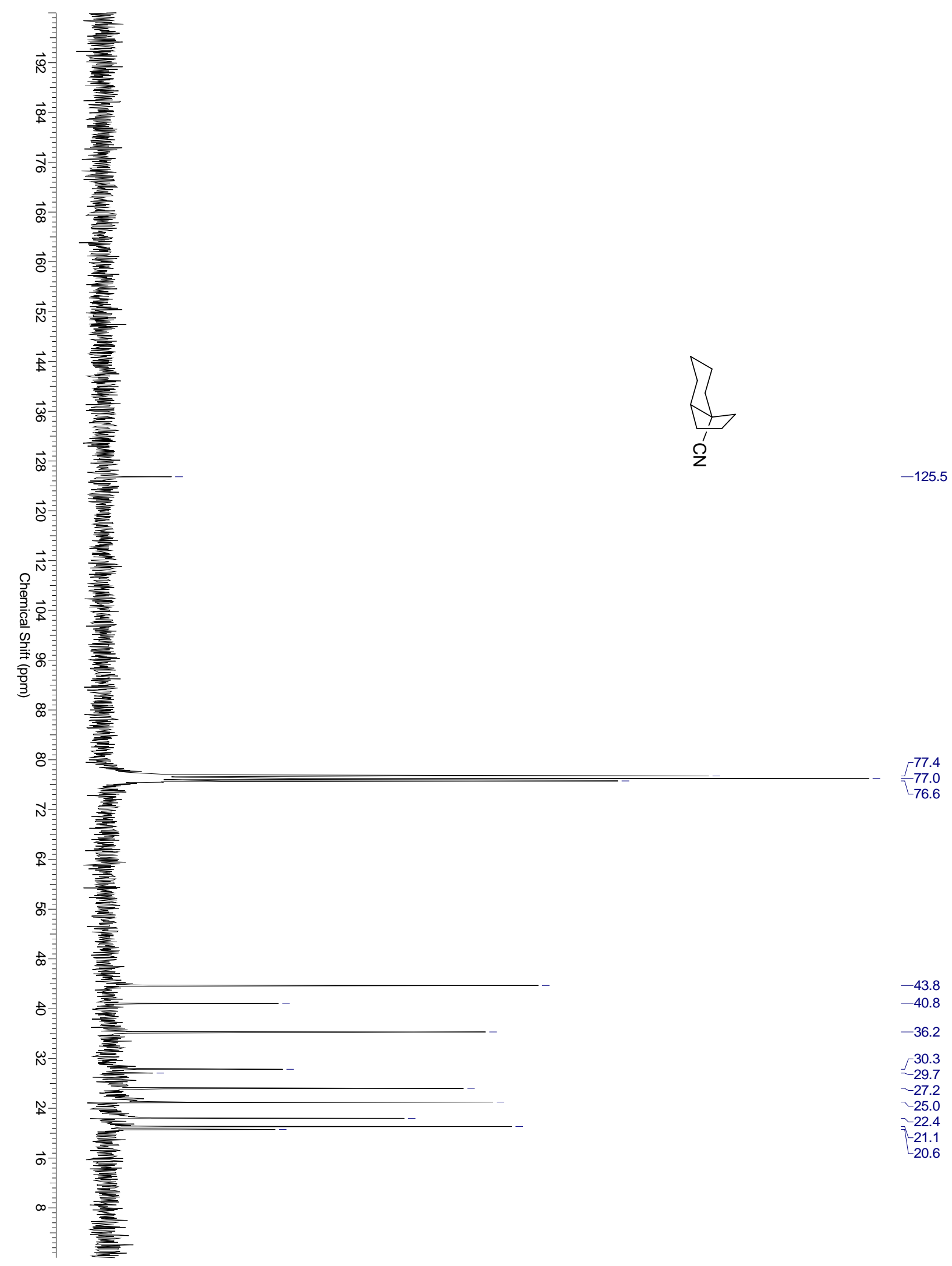




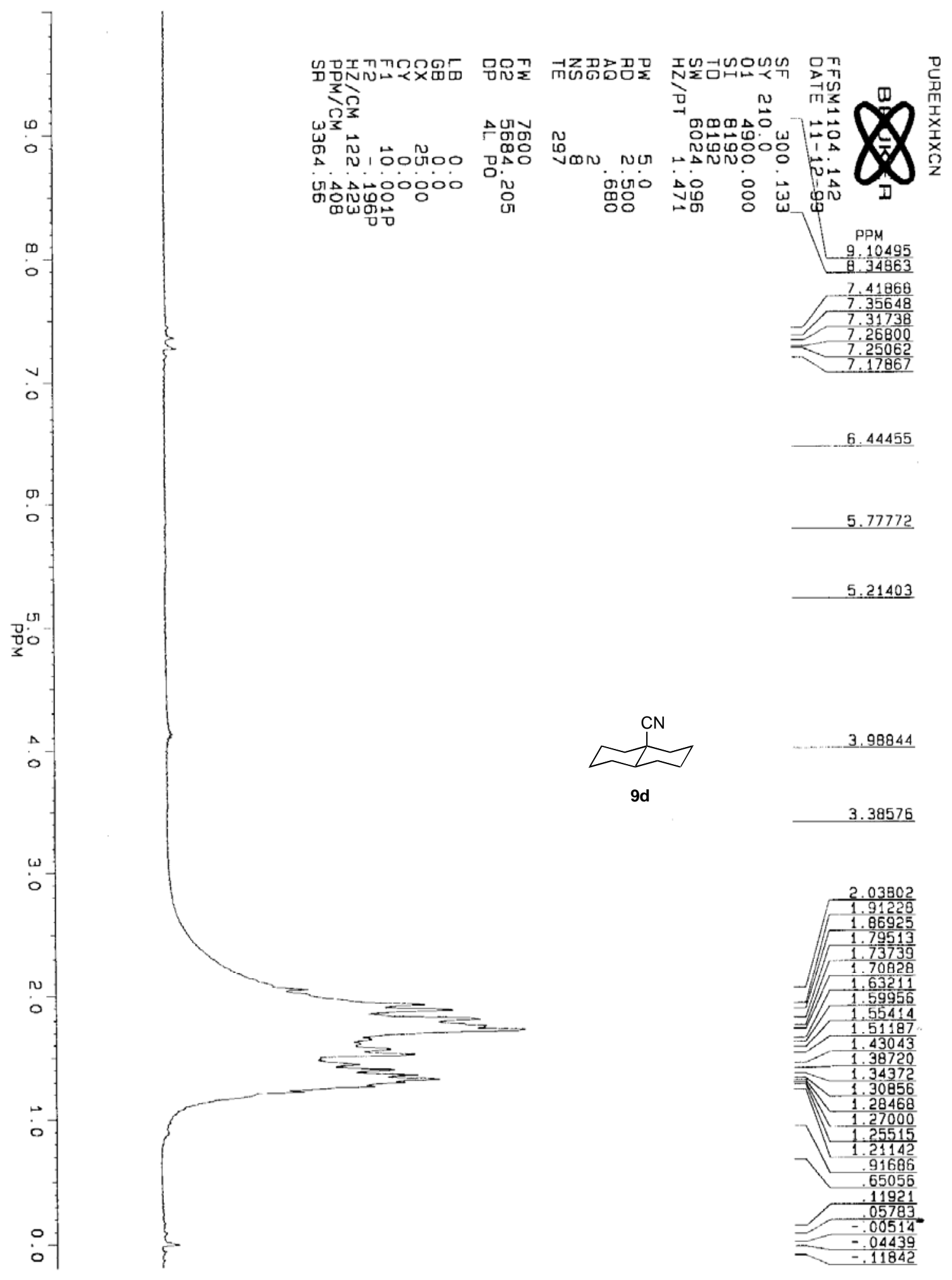




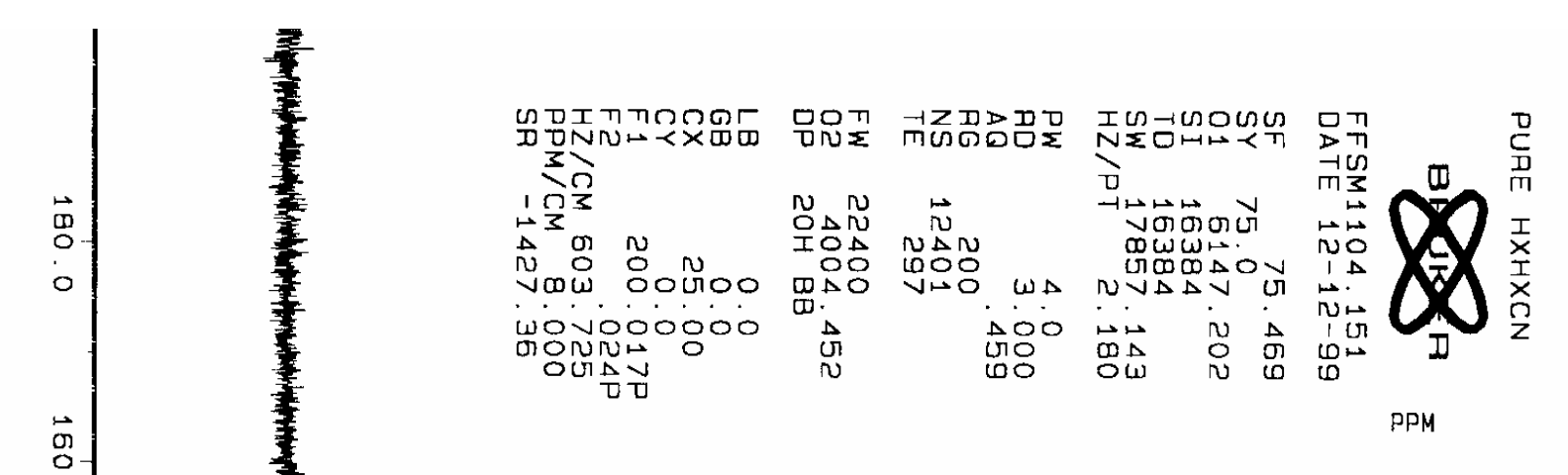

122. 839

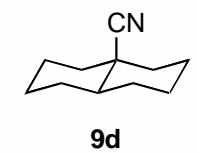

$\infty$
0
0

9

$\stackrel{\circ}{\circ}$
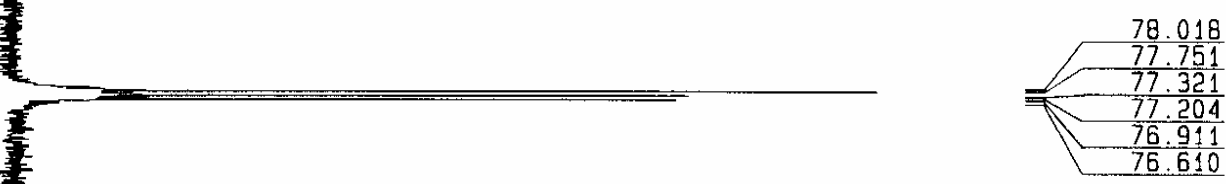

0
0

45.430

45.112

42.973

38.699

0

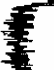

E

等

37.226

31.016

30.730

26.079 